\title{
UM MÉTODO PARA CONDUÇÃO DE INVENTÁRIOS FLORESTAIS SEM O USO DE EQUAÇÕES VOLUMÉTRICAS ${ }^{1}$
}

\author{
Helio Garcia Leite ${ }^{2}$ e Valdir Carlos Lima de Andrade ${ }^{3}$
}

\begin{abstract}
RESUMO - Neste trabalho foram conduzidos testes preliminares sobre o uso do método altura relativa, desenvolvido originalmente por Andrade \& Leite (1997), para condução de inventário florestal. O método proposto foi comparado com um dos métodos usuais, que consiste em medir o diâmetro de todas as árvores, a altura de cerca de 15 árvores e a altura de cerca de cinco árvores dominantes por parcela. Para processamento desses dados são utilizadas equações hipsométricas e volumétricas, sendo estas últimas geradas com dados de cubagem de árvores no povoamento. No método da altura relativa são utilizados apenas dados obtidos nas parcelas, não sendo necessário cubar árvores no povoamento. Os dados de quatro parcelas e de 277 árvores-amostra foram utilizados para comparação das duas metodologias (usual e altura relativa). Os volumes estimados pelos dois métodos foram iguais $\left(156,21\right.$ e 156,19 $\mathrm{m}^{3} / \mathrm{ha}$ ). Portanto, pode-se concluir que o método alternativo é promissor, resultando ainda em algumas vantagens, como: redução do número de alturas medidas nas parcelas e eliminação das atividades de cubagem de árvores-amostra e de ajuste de modelos volumétricos e, ou, de taper.
\end{abstract}

Palavras-chaves: Inventário florestal, método da altura relativa, taper e geometria analítica.

\section{A METHOD FOR CONDUCTING FOREST INVENTORIES WITHOUT USING VOLUMETRIC EQUATIONS}

\begin{abstract}
Preliminary tests were conducted of on the use of the Relative Height Method, developed originally by Andrade and Leite (1997), to corry out a forest inventory. The proposed method was compared with one of the conventional methods, that consists of measuring the diameter of all the trees, the height of about 15 trees and the height of about five dominant trees per plot. For processing these data, hipsometric and volumetric equations are used, with the latter being generated from tree scaling data in the stand. The relative height method includes only data obtained in the portions, without the need to scale trees in the stand. Data of four plots and 277 tree samples were used to compare the two methodologies (conventional and relative height). The volumes for the two methods were similar (156,21 and 156,19 $\mathrm{m}^{3} / \mathrm{ha}$ ). Thus, it can be concluded that the alternative method is promising, even resulting in some advantages, such as: reduction of the number of heights measured in the plots, elimination of the activities of tree sample scaling and adjustment of volumetric and, or, taper models.
\end{abstract}

Key words: $\quad$ Forest inventory, relative height method, taper and analytic geometry.

\section{INTRODUÇÃO}

A quantificação do volume sólido em povoamentos florestais é imprescindível para a implementação de planos de manejo sustentável das florestas. Para quantificar esse volume executa-se um inventário florestal que consiste na medição de parte da população, isto é, de unidades amostrais ou parcelas, para depois extrapolar

1 Recebido para publicação em 16.2.2000.

Aceito para publicação em 20.6.2002.

2 Prof. do Dep. de Engenharia Florestal da Universidade Federal de Viçosa - UFV, 36571-000 Viçosa-MG, <hgleite@mail.ufv.br>.

${ }^{3}$ Doutorando em Engenharia Florestal na Universidade Federal de Lavras - UFLA, Caixa Postal 37, 37200-000 Lavras-MG, <vclandrade@mailcity.com>. 
os resultados para a área total. Assim, visando planejar as operações florestais, têm-se estimativas da quantidade e da distribuição da madeira disponível.

Para obter o volume de cada parcela são utilizados modelos hipsométricos em conjunto com modelos volumétricos, de taper ou de múltiplos volumes. Assim, para obter as equações desses modelos, executa-se uma cubagem rigorosa em árvores-amostra abatidas. Esta cubagem, conforme FAO (1973), citada por Belchior (1996), é o método direto de estimação do volume de árvores mais utilizado na rotina de inventários florestais e consiste na medição sucessiva de diâmetros ao longo do tronco, dividindo-o em seções. A aplicação da fórmula de Smalian, por exemplo, resulta no volume sólido.

A atividade de cubagem rigorosa é feita em separado das medições das unidades amostrais, o que gera aumento no custo do inventário. Tal aumento pode ser reduzido se esta atividade for executada em medições simultâneas com as demais variáveis observadas, quando da medição das parcelas, contudo sem o abate das árvores. Com este intuito, estudou-se o método da altura relativa aplicado a tal condição. Neste método, apresentado em Andrade \& Leite (2001), para gerar equações de taper realizamse as medições na árvore em pé. Nesse caso, para estimar o volume não é necessário ajustar, analisar e, ou, testar equações volumétricas ou de taper, sendo exigidos apenas dados de diâmetro a 1,3 $\mathrm{m}$ do terreno (dap), da altura total $(H t)$ e do diâmetro em um ponto no intervalo entre dap e Ht. Além disto, esses dados são obtidos apenas para cerca de cinco árvores de cada parcela.

O objetivo deste estudo foi averiguar o comportamento do método da altura relativa, na condução de um inventário florestal, uma vez que é o cenário de desenvolvimento e uso deste método. Trata-se do primeiro teste desta metodologia, nesta situação. Não objetivou-se fazer inferências quanto ao nível de utilização da metodologia, em substituição aos métodos usuais de totalizar em volume sólido as parcelas de um inventário florestal.

\section{MATERIAL E MÉTODOS}

Os dados utilizados neste estudo foram provenientes de quatro parcelas circulares de $471,4 \mathrm{~m}^{2}$ de área, distribuídas casualmente em dois talhões com 67,8 ha implantados com eucalipto, tendo 7 anos de idade e conduzido em regime de alto fuste. A coleta dos dados foi feita por meio de duas metodologias, uma denominada de método usual e a outra de método da altura relativa.
O método usual é aquele adotado tradicionalmente por algumas empresas, que consiste na medição de $100 \%$ dos daps, de algumas Hts e da altura de cerca de cinco árvores dominantes. Neste caso, em cada parcela foram mensuradas as $\mathrm{Hts}$ das 15 primeiras árvores e das cinco árvores dominantes. Para estimar o volume utilizou-se o modelo de Schumacher \& Hall (1933), ajustado com os dados de uma cubagem rigorosa em 277 árvores-amostra abatidas. Os diâmetros ao longo do tronco foram medidos nas posições $0,1,0,3,0,7,1,3,2,4$, até $4 \mathrm{~cm}$ de diâmetro com casca.

No método da altura relativa foram medidos os daps de todas as árvores e a altura total de cinco árvores dominantes, e nas cinco primeiras árvores de cada parcela foram medidos a altura total e o diâmetro em uma altura entre 1,3m e Ht, definida por Andrade \& Leite (2001) como altura relativa $(h r)$. As alturas relativas foram obtidas pela tabela feita com a aplicação da equação deduzida do

modelo $\frac{d a p^{2} H t}{Y}=\beta_{0} d a p^{\beta_{1}}+\varepsilon \quad(1), \quad$ em que $Y=\frac{H t-h r}{H t}$. Este modelo foi ajustado com os dados das 277 árvores-amostra cubadas rigorosamente. As medições da $H t$, da altura relativa e do respectivo diâmetro (dhr) das cinco árvores de cada parcela foram feitas na árvore em pé, utilizando o Relascópio de Bitterlich. Ressalta-se que este instrumento foi utilizado apenas para realizar as medições já citadas, e não para propor um novo emprego deste. Poderia ser utilizado o Pentaprisma de Weeler, por exemplo.

Após as medições das parcelas selecionadas, pelo método usual e pelo método da altura relativa, todas as árvores foram abatidas e cubadas pelo método de Smalian, a fim de obter o volume real de madeira das parcelas. Esta cubagem foi realizada de maneira idêntica àquela feita nas 277 árvores-amostra.

A fim de realizar inferências sobre a exatidão das estimativas obtidas de volume até $4 \mathrm{~cm}$ de diâmetro com casca, foram estimadas as estatísticas porcentuais de desvio médio $\left(\right.$ DM $\left._{(\%)}\right)$, Bias $_{(\%)}$, correlação linear $\left({ }_{Y \hat{Y}_{(\%)}}^{r}\right)$ e erro-padrão da estimativa $\left({ }_{Y \hat{Y}_{(\%)}}\right)$, entre valores observados e estimados, precisão $\left(P_{\sigma_{\%}}\right)$, obtida pelo teste de qui-quadrado (Freese, 1960) e raiz quadrada do erro 
médio $\left(R Q E M_{(\%)}\right)$. A análise gráfica dos resíduos também foi utilizada para avaliar os dois métodos desenvolvidos. As fórmulas utilizadas no cálculo das estatísticas adotadas foram:

$$
\begin{aligned}
& D M_{(\%)}=\frac{1}{n} \sum_{i=1}^{n}\left(\frac{\hat{Y_{i}}-Y_{i}}{Y_{i}}\right) 100 \\
& \operatorname{Bias}(\%)=\frac{\left(\sum_{i=1}^{n} \hat{Y}_{i}-\sum_{i=1}^{n} Y_{i}\right)}{\sum_{i=1}^{n} Y_{i}} 100 \\
& r_{Y \hat{Y}(\%)}=\left[\frac{\sum_{i=1}^{n} Y_{i} \hat{Y}_{i}-\frac{\left(\sum_{i=1}^{n} Y_{i}\right)\left(\sum_{i=1}^{n} \hat{Y}_{i}\right)}{n}}{\sqrt{\left\{\left[\sum_{i=1}^{n}\left(Y_{i}-\bar{Y}\right)^{2}\right]\left[\sum_{i=1}^{n}\left(\hat{Y}_{i}-\bar{Y}\right)^{2}\right]\right\}}}\right] 100 \\
& S_{Y \hat{Y}(\%)}=\left[\frac{\sqrt{\sum_{i=1}^{n}\left(Y_{i}-\hat{Y}_{i}\right)^{2}} /(n-p-1)}{\bar{Y}}\right] 100 \\
& P_{\%}=\sqrt{\left[\frac{196^{2}}{\chi_{n}^{2}} \sum_{i=1}^{n}\left(\frac{Y_{i}-\hat{Y_{i}}}{Y_{i}}\right)\right]} \\
& \operatorname{RQEM}_{(\%)}=\left[\frac{\sqrt{\frac{\sum_{i=1}^{n}\left(Y_{i}-\hat{Y}_{i}\right)^{2}}{n}}}{\bar{Y}}\right]
\end{aligned}
$$

em que

$\hat{Y}_{i}$ e $Y_{i}=$ volume estimado e observado até o diâmetro comercial de $4 \mathrm{~cm}$, respectivamente, $\mathrm{em} \mathrm{m}^{3}$;

$n=$ número de pares de $\hat{Y}_{i}$ e $Y_{i}$; $p=$ número de parâmetros da regressão linear simples entre $Y_{i}$ e $\hat{Y_{i}}$

$\bar{Y}=$ volume médio observado até o diâmetro comercial de $4 \mathrm{~cm}$ em m${ }^{3}$; e

$P_{\%}=$ precisão em porcentagem; 1 ,96 é o valor do desviopadrão normal para probabilidade bilateral de 5\%, e

$\chi_{n}^{2}$ é o valor tabelado do qui-quadrado para $n$ graus de liberdade.

Os resultados das estatísticas adotadas foram totalizados para classificar as estimativas obtidas. A estatística $r_{Y \hat{Y}_{(\%)}}$ foi transformada para $100-r_{Y \hat{Y}(\%)}$, visando manter o critério do total, ou seja, quanto menor o valor melhor o resultado obtido pelo método utilizado (Andrade, 2001). Esse total porcentual foi obtido por:

$\operatorname{total}_{\%}=D M_{(\%)}+\operatorname{Bias}_{(\%)}+\left(100-r_{Y \hat{Y}_{(\%)}}\right)+s_{Y \hat{Y}_{(\%)}}+P_{\%}+R Q E M_{(\%)}$

\section{RESULTADOS E DISCUSSÃO}

\subsection{Método da Altura Relativa}

$$
\begin{aligned}
& \text { Após o ajuste do modelo } 1 \text { obteve-se } \\
& \frac{d a p^{2} H t}{(H t-h r) / H t}=11,963048 d a p^{2,523202}, \text { com } \bar{R}^{2}=94,59 \% \mathrm{e}
\end{aligned}
$$
n=277 árvores-amostra. A partir desta equação, deduziuse que:

$$
\begin{aligned}
& d a p^{2} H t=\left(\frac{H t-h r}{H t}\right)\left(11,963048 d a p^{2,523202}\right) \\
& \frac{H t-h r}{H t}=\frac{d a p^{2} H t}{11,963048 d a p^{2,523202}} \\
& H t-h r=H t\left(\frac{d a p^{2} H t}{11,963048 d a p^{2,523202}}\right) \\
& \hat{h r}=H t-H t\left(\frac{d a p^{2} H t}{11,963048 d a p^{2,523202}}\right) \\
& \hat{h r}=H t-H t\left(11,963048^{-1} d a p^{-2,523202} d a p^{2} H t\right) \\
& \hat{h r}=H t-H t^{2} \quad 0,083591 d a p^{-0,523202}
\end{aligned}
$$


A equação 2 foi utilizada para estimar a altura relativa em condições de campo, tendo sido construída a tabela apresentada no Quadro 1. Utilizando essa tabela, coletaram-se os dados pelo método da altura relativa, nas quatro parcelas. Em seguida, foram empregadas as informações obtidas das cinco árvores medidas em cada parcela (dap, $H t, h r$ e $d h r)$ no cálculo do coeficiente angular da reta que é formada nos intervalos entre o dap e a altura relativa (intervalo I) e entre a altura relativa e a altura total (intervalo II). Estes coeficientes angulares $(C A R)$ foram obtidos, conforme Andrade \& Leite (2001), por:

$$
\begin{aligned}
C A R_{I} & =\frac{1,3-h r}{(d a p-d h r) / 2} \\
C A R_{I I} & =\frac{h r-H t}{(d h r) / 2}
\end{aligned}
$$

em que

$h r=$ altura relativa, em $\mathrm{m} ;$

$d h r=$ diâmetro medido na altura $h r, \mathrm{em} \mathrm{cm}$; e

as demais variáveis foram definidas anteriormente.

Após os cálculos foram ajustados os modelos de regressão propostos por Andrade \& Leite (1998b), obtendo-se as equações:

$$
\begin{aligned}
& \left(\frac{d a p^{2} H t}{C A R_{I}}\right)=-0,075186 d a p^{3,612660} \\
& \left(\frac{d a p^{2} H t}{C A R_{I I}}\right)=-2,094968 d a p^{2,584254}
\end{aligned}
$$

com coeficientes de determinação ajustados $\left(\bar{R}^{2}\right)$ de $87,24 \%$ e $88,45 \%$, respectivamente.

A partir das equações 5 e 6 deduziu-se que:

$$
\begin{aligned}
& \hat{C A R_{I}}=\frac{d a p^{2} \cdot H t}{-0,075186 \cdot d a p^{3,612660}} \\
& \hat{d}_{\left(h r<h_{i} \leq H t\right)}=\frac{d a p^{1,612660}}{H t}\left(0,097742-0,075186 h_{i}\right)+\frac{d a p^{0,584254}}{H t}\left(2,094968 H t-2,094968 h_{i}\right)+0,5 d a p
\end{aligned}
$$

$$
\hat{C A R_{I I}}=\frac{d a p^{2} \cdot H t}{-2,094968 \cdot d a p^{2,584254}}
$$

As equações 7 e 8 , após serem substituídas nas equações 3 e 4, respectivamente, geraram as equações de taper. Assim, fazendo $h r=h_{i}$ e $d h r=d_{I}$ na equação 3 e $d h r=d_{I I}$ na equação 4, com $h r=h_{i}$, deduz-se que:

$$
\begin{aligned}
& \frac{d a p-d_{I}}{2}=\frac{1,3-h_{i}}{\left(\frac{d a p^{2} \cdot H t}{-0,075186 \cdot d a p^{3,612660}}\right)} \\
& \hat{d_{I}}=\frac{d a p^{1,612660}}{H t}\left(0,195484-0,150372 h_{i}\right)+d a p(9) \\
& \frac{d_{I I}}{2}=\frac{h_{i}-H t}{\left(\frac{d a p^{2} H t}{-2,094968 d a p^{2,584254}}\right)} \\
& \hat{d}_{I I}=\frac{d a p^{0,584254}}{H t}\left(2,094968 H t-2,094968 h_{i}\right)
\end{aligned}
$$

em que

$$
\hat{d}_{I}=\text { diâmetro com casca estimado pela equação }
$$
obtida por meio do coeficiente angular da reta formada pelo intervalo I (entre $1,3 \mathrm{~m}$ e $h r$ );

$$
\hat{d}_{I I}=\text { diâmetro com casca estimado pela equação }
$$

obtida por meio do coeficiente angular da reta formada pelo intervalo II (entre $h r$ e $H t$ ); e

$$
h_{i}=\text { altura desejada, } \mathrm{m} \text {. }
$$

As equações de taper 9 e 10 são utilizadas para estimar diâmetros ao longo do tronco, simulando-se uma cubagem rigorosa. Para obter o volume aplica-se a fórmula de Smalian (Andrade \& Leite, 1998a, 2001). Desta forma, emprega-se a equação 9 em posições loca- 
Quadro 1 - Tabela utilizada para obter a altura relativa em situações de campo, gerada por meio de $\hat{h r}=H t-H t^{2} \quad 0,083591$ dap $^{-0,52320229}$

Table 1 - Table utilized to obtain relative height, under field conditions, generated by means of: $\hat{h r}=H t-H t^{2} \quad 0,083591 d a p^{-0,52320229}$

\begin{tabular}{|c|c|c|c|c|c|c|c|c|c|c|c|}
\hline \multirow{2}{*}{\multicolumn{2}{|c|}{ Classe Ht (m) }} & \multicolumn{10}{|c|}{ Classe de dap $(\mathrm{cm})$} \\
\hline & & \multirow{2}{*}{$\begin{array}{c}5,1 \text { a } 7,0 \\
6 \\
5,9\end{array}$} & \multirow{2}{*}{$\begin{array}{c}7,1 \text { a } 9,0 \\
8 \\
6,2\end{array}$} & \multirow{2}{*}{$\begin{array}{c}9,1 \text { a } 11,0 \\
10 \\
6,4\end{array}$} & \multirow{2}{*}{\begin{tabular}{|c|}
11,1 a 13,0 \\
12 \\
6,5
\end{tabular}} & \multirow{2}{*}{$\begin{array}{c}13,1 \text { a } 15,0 \\
14 \\
6,7\end{array}$} & \multirow{2}{*}{$\begin{array}{c}15,1 \text { a } 17,0 \\
16 \\
6,7\end{array}$} & $\begin{array}{c}17,1 \text { a } 19,0 \\
18\end{array}$ & $\begin{array}{c}19,1 \text { a } 21,0 \\
20\end{array}$ & $\begin{array}{c}21,1 \text { a } 23,0 \\
22\end{array}$ & $\begin{array}{c}23,1 \text { a } 25,0 \\
24 \\
\end{array}$ \\
\hline 7,1 a 9,0 & 8 & & & & & & & & & & \\
\hline 9,1 a 11 & 10 & 6,7 & 7,2 & 7,5 & 7,7 & 7,9 & 8,0 & & & & \\
\hline 11,1 a 13 & 12 & 7,3 & 7,9 & 8,4 & 8,7 & 9,0 & 9,2 & 9,3 & & & \\
\hline 13,1 a 15 & 14 & 7,6 & 8,5 & 9,1 & 9,5 & 9,9 & 10,2 & 10,4 & 10,6 & & \\
\hline 15,1 a 17 & 16 & 7,6 & 8,8 & 9,6 & 10,2 & 10,6 & 11,0 & 11,3 & 11,5 & 11,8 & 11,9 \\
\hline 17,1 a 19 & 18 & 7,4 & 8,9 & 9,9 & 10,6 & 11,2 & 11,7 & 12,0 & 12,4 & 12,6 & 12,9 \\
\hline 19,1 a 21 & 20 & 6,9 & 8,7 & 10,0 & 10,9 & 11,6 & 12,2 & 12,6 & 13,0 & 13,4 & 13,7 \\
\hline 21,1 a 23 & 22 & 6,2 & 8,4 & 9,9 & 11,0 & 11,8 & 12,5 & 13,1 & 13,6 & 14,0 & 14,3 \\
\hline 23,1 a 25 & 24 & & 7,8 & 9,6 & 10,9 & 11,9 & 12,7 & 13,4 & 14,0 & 14,4 & 14,9 \\
\hline
\end{tabular}

\subsection{Quantificação do Volume de Madeira das Quatro Parcelas e Análise dos Resultados}

A medição das parcelas foi feita pelo método usual e pelo método da altura relativa. Na totalização das parcelas, usando o método usual, foram ajustadas uma equação hipsométrica e outra volumétrica, que resultaram em:

$$
\begin{aligned}
& \hat{H} t=e^{\left(3,604094-\frac{7,653112}{d a p}\right)} \\
& \bar{R}^{2}=76,1 \% \text { e } \mathrm{n}=60 \text { pares de } H t \text { e dap } \\
& \hat{V}=e^{-10,648633+1,835865} \cdot{ }^{\text {Ln}(\text { dap })+1,254373} \cdot{ }^{L n(H t)} ; \\
& \bar{R}^{2}=99,4 \% \text { e } \mathrm{n}=277 \text { árvores-amostra cubadas }
\end{aligned}
$$

em que

$\hat{H} t=$ altura total estimada; $L n=$ logaritmo neperiano.

Na totalização das parcelas, quando do emprego do método da altura relativa, usou-se a altura total estimada por uma equação ajustada com os valores das cinco árvores medidas por parcela. A equação hipsométrica obtida foi:

$$
\begin{aligned}
& \hat{H} t=e^{3,792684-11,123730 \cdot \frac{1}{d a p}} ; \\
& \bar{R}^{2}=79,54 \% \text { e } \mathrm{n}=20 \text { pares de } H t \text { e dap. }
\end{aligned}
$$

Após totalizado o volume até $4 \mathrm{~cm}$ de diâmetro, procedeu-se à averiguação das estimativas proporcionadas pelos métodos utilizados. Foram utilizados a análise de resíduos e o resultado do total $_{\%}$, apresentados nas Figuras 1 e 2 .

Pela análise gráfica, Figuras 1 e 2, verifica-se que as metodologias comparadas apresentam uma semelhança na tendência das estimativas, sendo melhor para o método usual. Também, este método apresentou um total porcentual menor que o obtido pelo método da altura relativa $(49,4 \%$ contra $49,5 \%)$.

Cabe ressaltar que, em geral, o modelo de Schumacher $\&$ Hall (1933) resulta em estimativas precisas e livres de tendência. Os resultados das Figuras 1 e 2 indicam tendenciosidade, porém os volumes comparados nessas figuras são oriundos apenas de quatro parcelas. Em relação ao objetivo do trabalho, o importante é que a tendência observada é a mesma, podendo-se concluir que o procedimento desenvolvido por Andrade \& Leite (1997) é promissor. Pode-se observar ainda (no 
Quadro 2) que os volumes estimados por hectare com os dois métodos são iguais.

Considerando que o resultado das análises evidenciou a possibilidade de obter estimativas aproximadamente iguais, então há evidências de estimativas promissoras pelo emprego do método da altura relativa na condução de inventários florestais. Além de resultados promissores, o emprego da metodologia proposta mostra haver evidências de:

1. reduzir a medição de alturas totais por parcela, para desenvolver modelos hipsométricos. Neste trabalho, das 15 primeiras árvores reduziu-se para 5;

2. permitir a redução do número de árvores-amostra cubadas. Observou-se que é possível medir seis árvores em pé com o método da altura relativa no tempo que se gastaria, com o método usual, para cubar uma única árvore abatida;

3. permitir a coleta de todas as informações necessárias simultaneamente com a medição das parcelas. No método usual, a atividade cubagem é feita em separado, acarretando o abate de árvores-amostra; e

4. permitir a condução de estudos de diferentes usos da árvore, sem a necessidade de uma equação específica para estimar as alturas ao longo do tronco (taper), uma vez que a fórmula de Smalian é usada em uma cubagem rigorosa estimada.

A partir dessas considerações, pode-se inferir que o método da altura relativa é recomendável para conduzir inventários florestais. Assim, o método de inventário florestal proposto consiste em coletar dados apenas nas parcelas de inventário, excluindo o abate de árvoresamostra e o ajuste de modelos volumétricos e, ou, de taper.

Todavia, as equações de taper geradas pelo método da altura relativa parecem ser uma proposta sem ganhos estatísticos, devendo-se, então, preferir a condução de um inventário florestal adotando o método usual. Este tipo de questionamento se agrava ainda mais ao ser considerado o fato de que a metodologia proposta ainda depende de dados de árvores-amostra cubadas previamente, a fim de obter uma equação para calcular a altura relativa $h r$, cuja posição é determinada para medir o diâmetro $d h r$ em situações de campo, com a árvore em pé.

Métodos de determinação da $h r$, sem o uso de dados de cubagem e ajuste de modelos de regressão, já foram desenvolvidos por Andrade \& Leite (1998a). Ainda, é importante testar outras divisões na árvore para efeito

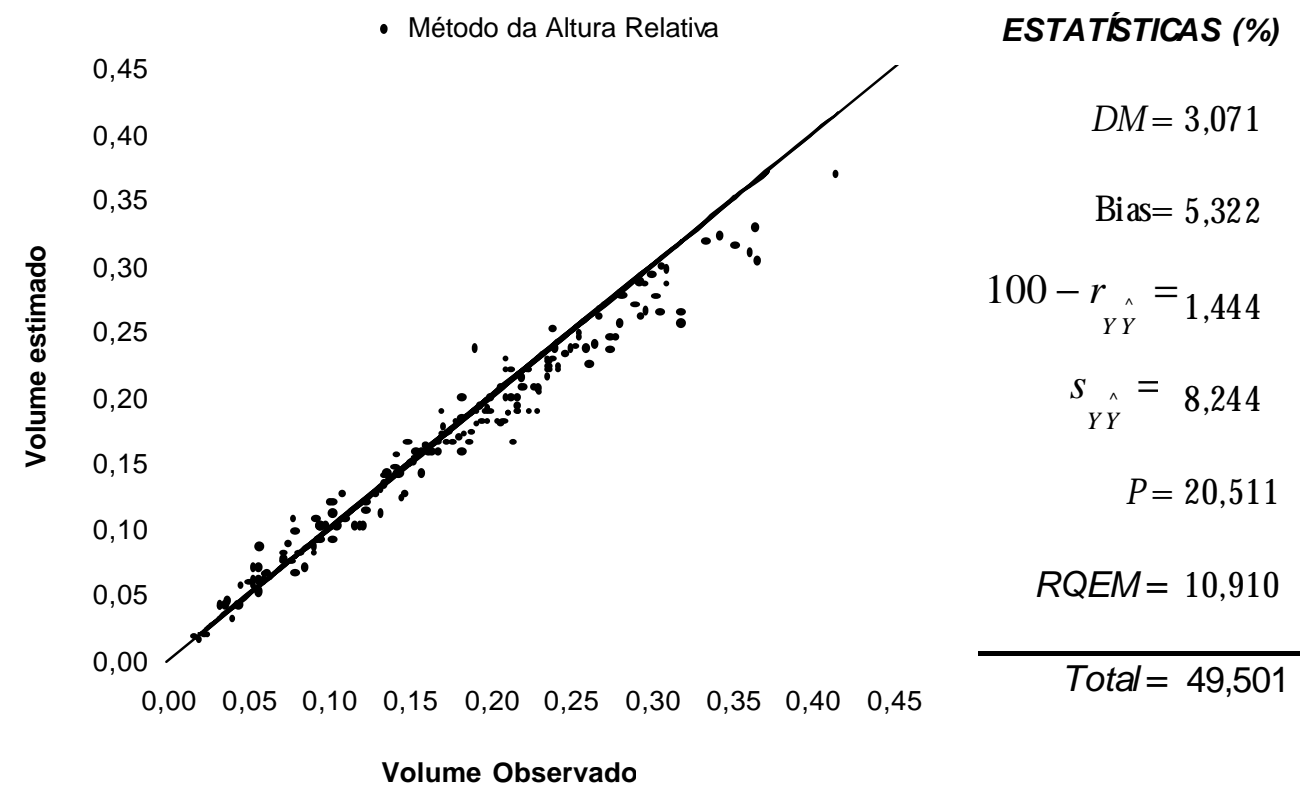

Figura 1 - Volume estimado até $4 \mathrm{~cm}$ de diâmetro em relação do volume observado, utilizando o método altura relativa. Figure 1 - Volume estimated up to $4 \mathrm{~cm}$ of diameter in relation to the observed volume, using the relative height method. 


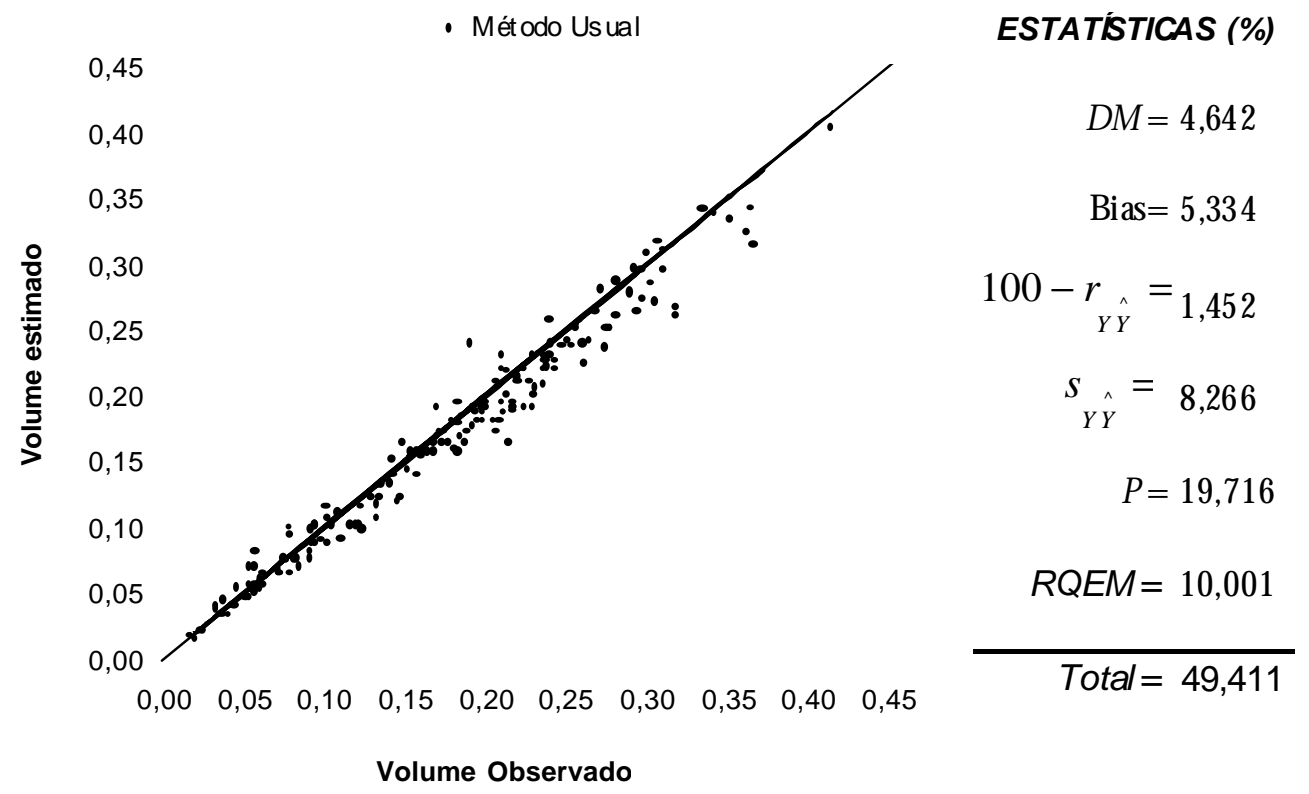

Figura 2 - Volume estimado até $4 \mathrm{~cm}$ de diâmetro em relação ao volume observado, utilizando o método usual.

Figure 2 -Volume estimated up to $4 \mathrm{~cm}$ of diameter in relationship to the observed volume, using the conventional method.

Quadro 2 - Estatísticas do inventário florestal Table 2 - Forest inventory statistics

\begin{tabular}{|c|c|c|c|c|c|c|}
\hline \multirow{2}{*}{ Método } & \multirow{2}{*}{$\bar{V}_{h a}\left(\mathrm{~m}^{3} / \mathrm{ha}\right)$} & \multirow{2}{*}{$C V_{\%}$} & \multirow{2}{*}{$S_{V}\left(\mathrm{~m}^{3} / \mathrm{ha}\right)$} & \multirow{2}{*}{$\varepsilon_{\%}$} & \multicolumn{2}{|c|}{ Limite de Confiança $\left(\mathrm{m}^{3} / \mathrm{ha}\right)$} \\
\hline & & & & & Inferior & Superior \\
\hline Real & 164,99 & 7,32 & 6,035 & 11,64 & 145,79 & 184,20 \\
\hline Altura relativa & 156,21 & 7,64 & 5,967 & 12,16 & 137,22 & 175,20 \\
\hline Usual & 156,19 & 8,54 & 6,667 & 13,58 & 134,97 & 177,41 \\
\hline
\end{tabular}

em que $\bar{V}_{h a}=$ volume médio por ha, $\mathrm{CV}_{\%}=$ coeficiente de variação, $S_{V}=$ desvio-padrão da média e $\varepsilon_{\%}=$ erro de amostragem.

do cálculo do coeficiente angular da reta e, assim, melhorar as estimativas do taper. Este estudo também já foi feito e é apresentado em Andrade (2001).

Quanto à cubagem, ressalta-se que esta atividade ainda deverá persistir no setor florestal, pois é prudente averiguar os resultados obtidos pelo emprego do método aqui apresentado. O importante é salientar que não será mais necessário realizar a cubagem de árvores-amostra com o compromisso de atualizar o banco de equações para adequar à situação encontrada, quando da condução de um inventário florestal. Tal procedimento é muito útil, uma vez que se já existe um banco de dados de cubagem disponível pode-se, então, aproveitá-lo para definir o melhor procedimento a ser adotado no desenvolvimento do método proposto, e após algumas averiguações ao longo do tempo serão obtidos resultados satisfatórios ao ponto de optar em não mais realizar a cubagem para povoamentos florestais de determinada característica.

A argumentação mais relevante para recomendação do método da altura relativa, em substituição aos métodos tradicionais de conduzir inventários florestais, é que existe uma real possibilidade de viabilizar a medição das variáveis, necessárias ao desenvolvimento do método proposto, simultaneamente com a medição das parcelas. Tal procedimento é importante, principalmente pelo fato de que, às vezes, a cubagem pode não representar toda a 
área inventariada. Com a proposta, representa-se a mesma área percorrida pelas parcelas do inventário e, assim, todo evento que ocorrer na área estará sendo representado pelas parcelas e também pelas equações de taper geradas, ou seja, estará aproveitando-se da aleatoriedade proporcionada pela distribuição das parcelas na área florestal inventariada.

Como ilustração, foram incluídas, no Quadro 2, algumas estatísticas do inventário conduzido por meio do método usual e do método proposto. Foram incluídas ainda as estatísticas obtidas, utilizando os volumes reais das quatro parcelas.

Ao analisar os resultados do Quadro 2, verifica-se que os volumes estimados pelos dois métodos são iguais. Obviamente, para reduzir o erro de amostragem, em ambos os casos, será necessário aumentar o número de parcelas. Ressalta-se que o objetivo principal deste trabalho foi testar o método altura relativa em um inventário florestal, e não indicar sua adoção definitiva em substituição ao método usual.

\section{CONCLUSÃO}

Diante dos resultados obtidos conclui-se que ao utilizar o método da altura relativa, em uma hipótese teórica, pode-se esperar resultados estatisticamente iguais àqueles obtidos pelo método usual, com as vantagens de reduzir o número de alturas totais amostradas, permitir a medição simultânea de todas as variáveis mensuradas em um inventário florestal e eliminar as atividades pertinentes ao ajuste de modelos volumétricos e, ou, de taper. Estas vantagens ocasionam maior agilidade na execução de um inventário florestal, com redução no custo, além de não prejudicar a qualidade das estimativas.

A medição de apenas cinco árvores por parcela, ao adotar o método da altura relativa para o povoamento utilizado, é suficiente.

\section{REFERÊNCIAS BIBLIOGRÁFICAS}

ANDRADE, V. C. L.; LEITE, H. G. Análise do perfil do tronco de árvores utilizando geometria analítica. Viçosa: Universidade Federal de Viçosa, 1997. 35 p. (Relatório não Publicado de Iniciação Científica Sem Bolsa de Pesquisa Graduação em Engenharia Florestal) - Universidade Federal de Viçosa, 1997.

ANDRADE, V. C. L.; LEITE, H. G. Um novo método para estimar volume de árvores individuais. In: SEMINÁRIO DE INICIAÇÃO CIENTÍFICA, 6, 1998, Ouro Preto. Resumos... Ouro Preto: UFOP, 1998a. p. 69-70.

ANDRADE, V. C. L.; LEITE, H. G. Uma nova metodologia para medir e totalizar parcelas em um inventário florestal. In: SEMINÁRIO DE INICIAÇÃO CIENTÍFICA, 6, 1998, Ouro Preto, Resumos... Ouro Preto: UFOP, 1998b. p. 73-74.

ANDRADE, V. C. L.; LEITE, H. G. Uso da geometria analítica para descrever o taper e quantificar o volume de árvores individuais. Revista Árvore, v. 25, n. 4, p.481-486, 1998c.

ANDRADE, V. C. L. Um método para descrever o perfil do tronco em árvores de eucalipto utilizando geometria analítica. Viçosa: Universidade Federal de Viçosa, 2001. 70 p. Dissertação (Mestrado em Ciência Florestal) Universidade Federal de Viçosa, 2001.

BELCHIOR, P. R. M. Estimação de volumes total, de fuste e de galhos em mata secundária no município de Rio Vermelho, MG. Viçosa: Universidade Federal de Viçosa, 1996. 75 p. Dissertação (Mestrado em Ciência Florestal) Universidade Federal de Viçosa, 1996.

FREESE, F. Testing acuracy. Forest Science, v. 6, n. 2, p. 139-145, 1960.

SCHUMACHER, F. X.; HALL, F. S. Logarithmic expression of the timber volume. Journal of Agriculture Research, v. 47, n. 9, p. 719-34, 1933. 